A. Weisbecker, M. Burmester \& A. Schmidt (Hrsg.): Mensch und Computer 2015 Workshopband, Stuttgart: Oldenbourg Wissenschaftsverlag, 2015, S. 595-604.

\title{
RehaInterAct - Der sensorbasierte Therapieraum der Zukunft
}

\author{
Anne Grohnert ${ }^{1}$, Anett Bölke ${ }^{3}$, Benny Häusler ${ }^{2}$, Boris Irmscher ${ }^{3}$, Michael \\ John $^{2}$, Andreas Kliem ${ }^{1}$, Gerd Kock ${ }^{2}$, Jens Piesk ${ }^{3}$, Marco Polak ${ }^{3}$ \\ Komplexe und Verteilte IT Systeme (CIT), Technische Universität Berlin ${ }^{1}$ Kompetenzzent- \\ rum E-HEALTH, Fraunhofer-Institut für Offene Kommunikationssysteme FOKUS ${ }^{2}$ \\ Nuromedia $\mathrm{GmbH}^{3}$
}

\section{Zusammenfassung}

Um Bewegungstherapien im häuslichen Umfeld zu ermöglichen, bedarf es ganzheitlicher Systeme, die sowohl den Trainierenden motivieren seinen Trainingsplan auch im Alltag regelmäßig einzuhalten als auch für medizinisches Personal die direkte Kontrolle über die Therapie zu gewährleisten. Das im Folgenden vorgestellte telemedizinische Assistenzsystem kann in der Rehabilitation und zur Prävention eingesetzt werden. Insbesondere in infrastrukturell schwachen Gebieten und bei Menschen, die in ihrer Beweglichkeit eingeschränkt sind, kann dieses System aufwendige Wege zu Therapie-Einrichtungen reduzieren. Durch die variabel gehaltene Integration von unterschiedlichster Sensorik, durch die medizinische Fernkontrolle und durch motivationale Aspekte aus dem Bereich des Serious Gaming kann das System für eine Vielzahl von Anwendungsfällen verwendet werden.

\section{$1 \quad$ Einleitung}

Schlaganfall ist eine der zentralen altersbedingten Krankheiten und erfordert oftmals eine langwierige Rehabilitation (Welsch 2015). Die Folgekosten durch Stürze bedingt durch Schlaganfall sind für das Gesundheitssystem sehr hoch. Verkürzte Liegezeiten im Krankenhaus führen dazu, dass der Patient in einem schlechteren Zustand in die stationäre Rehabilitation geht und oftmals nicht sofort mit dem belastungsintensiven Training zur Wiederherstellung der Beweglichkeit begonnen werden kann (Koczula et al. 2012). Hinzu kommt, dass sich die Zeiten des Aufenthalts in Reha-Einrichtungen ebenfalls verkürzt haben (Eiff \& Schüring 2011). Dies führt dazu, dass oftmals auch nach dem stationären Klinikaufenthalt noch Bedarf an Weiterversorgung und therapeutischem Bewegungstraining notwendig sind. Medizinische Rehabilitation muss als ein zusammenhängender Prozess verstanden werden, der nach dem Aufenthalt in Kliniken noch nicht abgeschlossen ist. 
Vielmehr geht es um eine begleitende Therapie, die ebenfalls Einzug in den alltäglichen Ablauf nehmen sollte, um nachhaltige Wirksamkeit zu gewährleisten (Aichner \& Holzer 2013). Insbesondere älteren Menschen, die oftmals eingeschränkt sind in der Bewegung, sind regelmäßige Besuche in Sport- und Therapieeinrichtungen unmöglich oder nur mit erheblichem Aufwand verbunden (Cyarto et al. 2008). Um Reha-Nachsorge im Alltag und somit auch im häuslichen Umfeld zu ermöglichen bedarf es ganzheitlicher Systeme, die gewährleisten, dass zum einen das rehabilitative Training in einem medizinisch adäquaten Rahmen stattfindet und sowohl behandelnder Arzt und Therapeut die medizinische Kontrolle über die Therapie auch über größere Entfernungen hinweg ausüben können als auch die Motivation des Patienten zu steigern, die Übungen seines Behandlungsplans regelmäßig auszuführen, ohne dass der Arzt zugegen ist (Hermens \& Vollenbroek-Hutten 2008). Systeme zum Überwachen von Vitalfunktionen im Medizinbereich und zum Trainieren im Fitnessbereich sind bereits etabliert und können auch mit entsprechenden Erweiterungen im Bereich der Rehabilitation eingesetzt werden. Mit Hilfe dieser Systeme können Patienten auf Basis von vom Arzt oder Therapeuten adaptierter Übungspläne sowohl im klinischen als auch im häuslichen Umfeld trainieren bei gewährleisteter medizinischer Kontrolle (John et al. 2013).

Um eine flächendeckende Versorgung zu gewährleisten, um Motivation zu schaffen und Kontrolle auch über Entfernungen hinweg zu gewährleisten, sind medizinische, auf Telematik basierende, ganzheitliche Systeme unumgänglich (Hamdi et al. 2014). Telemedizinische Assistenzsysteme erhöhen die Nachhaltigkeit der Reha-Nachsorge, da durch das Trainieren im häuslichen Umfeld eine Möglichkeit geschaffen wurde, die eigene Gesundheit orts- und zeitunabhängig zu fördern. Aufwendige Wege und damit einhergehende Kosten werden reduziert und somit das Gesundheitswesen patienten-zentrierter gestaltet (Körtke et al. 2012). Die Integration der Reha-Nachsorge in das Familienleben und somit in den Alltag ist als motivationaler Faktor nicht zu unterschätzen (Botella et al. 2009). Zudem können diese Systeme zur Prävention von Krankheiten eingesetzt werden.

Dieser Thematik haben sich unter anderem das Forschungsinstitut Fraunhofer FOKUS und die TU Berlin in Kooperation mit der Xybermind GmbH (Entwicklung der Sensorik), der Nuromedia GmbH (Visualisierung und Feedback Mechanismen) sowie dem Rehabilitationszentrum Lübben angenommen. In dem Projekt „RehaInterAct“ wird ein telemedizinisches Gesamtsystem entwickelt. Der Fokus liegt hierbei auf Schlaganfallpatienten. Diese sind in ihren Bewegungen meist stark eingeschränkt und müssen grundlegende Aktivitäten wie Laufen wieder neu erlernen. Dafür wurde unter anderem eine Gangschule entwickelt, welche durch das Assistenzsystem sowohl im klinischen als auch im häuslichen Umfeld eingesetzt werden kann. Im Folgenden wird dieses System vorgestellt. Kapitel 2 stellt die Systemarchitektur sowie die dazugehörigen Komponenten vor und in Kapitel 3 werden die Trainingsszenarien beschrieben, dargestellt durch Spiele aufgrund des motivationalen Aspekts.

\section{Systemarchitektur}

Um die im Projekt vorgesehenen Trainings-Szenarien im häuslichen Umfeld umsetzen zu können, bedarf es eines 3D-Therapieraums, in dem notwendige Sensorsysteme installiert 
sind. Auf Basis der sensorischen Daten können die Bewegungen analysiert und ausgewertet werden, sodass der Trainierende angeleitet werden kann, fehlerhafte Ausführungen sofort zu korrigieren. Zudem werden die Ergebnisse in aufbereiteter Form dem Therapeuten zugänglich gemacht. Zu diesem Zweck wurde das System auf die beiden Einheiten Therapie-Client und Therapie-Server aufgeteilt. Der Therapie-Client, integriert in den Therapieraum, setzt sich zusammen aus den Komponenten für die Sensor-Integration sowie Aggregation der sensorischen Daten, genannt Sensor-Aggregator und der Datenverarbeitung, welche aus der Bewegungsanalyse und einer interaktiven Benutzeroberfläche mit integrierten Feedbackmechanismen für den Patienten besteht. Der Therapie-Server befindet sich im zugeordneten Reha-Zentrum oder bei dem behandelnden Therapeuten und setzt sich zusammen aus einer Wissensbasis für die Integration der sensorischen Elemente, genannt Device Directory und der Therapeutenumgebung für die Verwaltung der Trainingspläne und der Patienten. Wenn ein Patient den Therapieraum nutzen will, so wird der Therapie-Client zunächst den aktuellen Übungsplan vom Therapie-Server abrufen, die notwendigen Sensoren auf Basis der vorhandenen Übungen ermitteln und das für die Integration notwendige Wissen aus dem Device Directory integrieren sowie nach der Durchführung der Übungen die Ergebnisse umgekehrt auf dem Therapie-Server speichern. Ein Therapeut kann diese Ergebnisse einsehen und bei Bedarf die entsprechenden Trainingspläne adaptieren und mit dem Patienten in Kontakt treten.

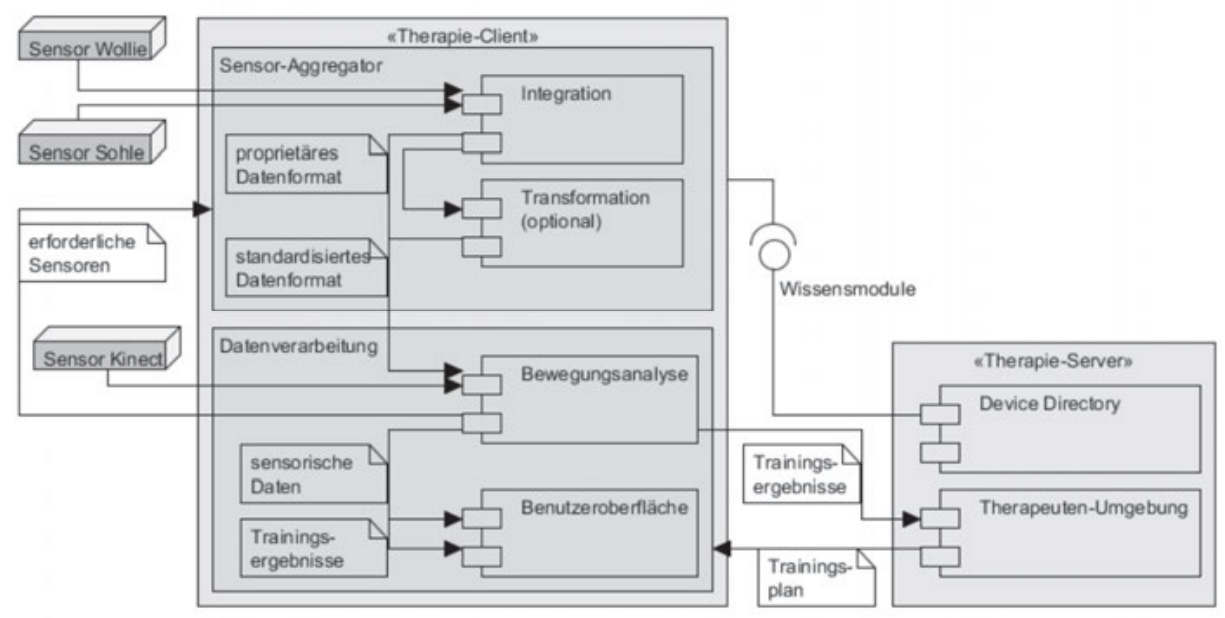

Abbildung 1: Systemarchitektur

\subsection{Sensoren}

Die verwendete Sensorik in dem hier beschriebenen telemedizinischen System bezieht sich auf zwei verschiedene Sensorknoten, die integriert in entsprechende Gegenstände sowohl für die Aktivierung der oberen als auch der unteren Extremitäten eingesetzt. Beide Sensorsyste- 
me setzen sich zusammen aus einer 9D-Bewegungssensorik (3D-Accelerometer, 3DGyroskop, 3D-Magnetometer) zur Bestimmung der Lage im Raum und FSR Drucksensoren. Integriert sind diese Sensorknoten zum einen in das Fußbett eines Hausschuhs als auch in ein neu entwickeltes Wand-Sensor-System, genannt Wollie-Action. Die Druckzonen der Schuhe sind aufgeteilt in die drei Bereiche lateral, medial und terminal, was bedeutet, dass für den seitlichen Fußbereich innen als auch außen sowie für den Fersenbereich unabhängige Belastungs- und Kraftintensitäten gemessen werden können. Die Wollie-Action besteht aus einem Schienensystem, in dem handflächen-große Pads verankert sind, die sowohl gedrückt, gezogen als auch verschoben werden können. Die verbaute Drucksensorik des Wandsystems kann neben der Druckkraft zudem auch durch einen neuartigen Mechanismus die Zugkraft messen. Die Übertragung der Bewegungs- und Druckdaten erfolgt über ein Bluegiga BT112 Funkmodul mittels Bluetooth 4.0 Datenprotokoll. Für eine verfeinerte Auswertung der Bewegungen wird zusätzlich ein optischer Sensor, in dem Fall die Kinect 1.0 von Microsoft, hinzugezogen und die 3D Skelettdaten per Kabel an den Therapieclient übertragen.

\subsection{Therapie-Client}

Der Therapie-Client setzt sich zusammen aus den beiden Komponenten Sensor-Aggregator sowie Datenverarbeitung und ist integriert in den 3D-Therapieraum, in dem das Bewegungstraining ausgeführt wird und somit die sensorischen Elemente angeordnet sind. In Hinblick auf eine Weiterentwicklung des Gesamtsystems sollen über die vorgestellten Trainingsszenarien hinaus auch weitere Übungen ermöglicht werden. Das führt dazu, dass unterschiedlichste Sensoriken benötigt werden. Durch die Heterogenität der Sensoren im Bereich der Funkund Kommunikations-Technologien sowie des genutzten Formats für die Datenübertragung wird je nach eingesetztem Gerät unterschiedliches Wissen für die Integration der Daten in das Gesamtsystem benötigt. Unterschiedliche Hersteller benutzen oft proprietäre Datenformate für die Kommunikation bei gleichartigen Geräten. Das führt dazu, dass bei Austausch eines Sensors durch einen anderen Hersteller auch die gesamten Daten-KommunikationsWege innerhalb eines Systems überarbeitet werden müssen. Das Management der Sensoren erfolgt daher über eine eigenständige Komponente, welche in der Lage ist, das notwendige Wissen für das Verwalten eines bestimmten Sensors zu ermitteln und dieses in Form von Modulen aus einem globalen Verzeichnis (i.e. Device Directory) dem System zur Verfügung zu stellen (Kliem et al. 2014). Um Homogenität der Daten sowie semantische Interoperabilität unabhängig vom Sensorhersteller für nachgeschaltete Komponenten wie der Datenverarbeitung zu gewährleisten, werden die sensorischen Daten gemäß der Normenfamilie ISO/IEEE 11073 übersetzt und in diesem standardisiertem Format weitergegeben (ISO/IEC/IEEE 2010).

Auf Basis der sensorischen Daten werden die medizinischen Bewegungs-Parameter der einzelnen Trainingsszenarien bewertet. Die unterschiedlichen Druckpartien der Schuhe ermöglichen die Analyse der Abrollbewegungen der Füße. Die richtige Reihenfolge der gesetzten Schritte und der Zeitabstände dazwischen gibt Auskunft über die Koordinationsfähigkeit. Während der Gangschule führt der Trainierende ein Pad der Wollie-Action mit sich. Besteht vollständiges Gleichgewicht, wird das Pad nicht als Stütze gebraucht, da weder Druck noch Zug ausgeübt wird. Ähnliche Verfahren werden bei Übungen zur Aktivierung der oberen 
Extremitäten eingesetzt. Durch Ziele innerhalb des Trainings-Szenariums wird der Patient aufgefordert, die Pads der Wollie-Action zu belasten und in bestimmte Richtungen zu verschieben. Auch hierbei können medizinische Parameter wie Koordination, Belastungsintensität und Beweglichkeit bewertet werden. Die optische Sensorik trägt dazu bei, die Körperhaltung zu kontrollieren, sodass eventuelle verletzende Ausführungen verhindert werden. Für eine sofortige Korrektur wird dem Trainierenden in Echtzeit der Fehler in der Ausführung insbesondere durch Sprache und zudem durch farbliche Hinweise ähnlich einem Ampelsystem in der Visualisierung mitgeteilt. Die aufbereiteten Ergebnisse einer Trainingseinheit werden remote an die Therapeuten-Umgebung übermittelt.

\subsection{Therapie-Server}

Der Therapie-Server besteht aus den beiden Komponenten Device Directory und Therapeuten-Umgebung und befindet sich im zugeordneten Reha-Zentrum. Das Device Directory fungiert als eine globale Wissensbasis und stellt das Wissen für die Verwaltung der benötigten Sensorik in Form von kleinen Modulen zur Verfügung. Diese variieren je nach Hersteller, Gerätetyp, verwendeter Funk- und Kommunikations-Technologie sowie Datenformat. Der Sensor-Aggregator lädt die notwendigen Wissenseinheiten nach feststellen des Sensortyps herunter und integriert diese autark, sodass das Gerät und die sensorischen Daten genutzt werden können. Wird der Sensor nicht mehr benötigt, werden die Module wieder entfernt. Somit kann bei Einführung eines neuen sensorischen Elements dieses sofort ohne manuelle, oft aufwendige Konfiguration genutzt werden.

Die Oberfläche der Therapeuten-Umgebung ermöglicht dem behandelnden Arzt oder Therapeuten den Zugriff auf die Daten, den Trainingsplan sowie die Ergebnisse des Trainings. Gegebenenfalls kann dort der Trainingsplan den Ergebnissen entsprechend unter medizinischer Kontrolle angelegt und modifiziert werden. Zudem können Arzt und Patient miteinander in Kontakt treten. Es können Termine durch ein Nachrichtensystem vereinbart werden oder auch durch Video-Telefonie in Echtzeit über weite Strecken hinweg miteinander gesprochen werden.

\section{Trainingsszenarien}

Durch eine hohe Varietät unterschiedlicher Krankheitsbilder gibt es eine Vielzahl von medizinischen Parametern, die innerhalb des Bewegungstrainings bewertet und analysiert werden müssen um falsche bzw. verletzende Ausführung zu vermeiden. Im Rahmen des Projektes wird eine Kategorisierung anhand der adressierten medizinischen Anwendungsfelder und der dafür geeigneten eingesetzten Sensorik vorgenommen. Es wird zwischen Freistehenden Übungen und Übungen am Wandschienensystem Wollie-Action unterschieden. Innerhalb dieser Kategorien sind verschiedene Trainingsszenarien definiert, die je nach Anwendungsfall individuell einstellbare Parameter besitzen. Um den Patienten zu motivieren, das Training auch im häuslichen Umfeld fortzuführen, werden die Trainingsszenarien durch Spiele dargestellt. Zu jeder Übung wurde ein Spielszenario konzipiert, das die Bewegungsabläufe 
der jeweiligen Übung bestmöglich spielerisch abbildet. So wurde unter anderem für die Gangschule in der Kategorie Freistehende Übungen das Spielszenario Überqueren eines Baches über Steine und für Übungen am Wandschienen-System das Spiel Drachenfliegen entwickelt. Im Folgenden werden die Schwerpunkte beider Kategorien und die Bewertung der medizinischen Parameter anhand der eingesetzten Sensorik sowie die Umsetzung dessen exemplarisch an jeweils einem Spielszenario beschrieben.

\subsection{Freistehende Übungen}

Diese Trainingseinheiten werden insbesondere genutzt zur Aktivierung der unteren Extremitäten, z. B. durch eine Gangschule. Nach einem Schlaganfall muss oftmals das Gehen mit dem nötigen Gleichgewicht neu erlernt werden. Zur Messung der medizinischen Parameter wie die richtige Abrollbewegung, gleichmäßige Druckverteilung beider Füße zueinander und der Schrittlänge sowie der Koordination und des Gleichgewichts werden sowohl die Schuhsensorik als auch die Wollie-Action eingesetzt. Durch die Druck- und Lage-Sensorik der Schuhe kann die Art und Belastungsintensität des Auftretens ausreichend bewertet werden. Die Wollie-Action gibt Auskunft über das Vorhandensein des Gleichgewichts, in dem gegebenenfalls Druck bzw. Zugkraft, ausgeübt durch den Trainierenden, gemessen wird. Um auch eine achsengerechte Oberkörperaufrichtung und axiale Beinstellung beurteilen zu können und somit falsches, ggf. sogar verletzendes Ausführen zu verhindern, wird zudem die optische Sensorik genutzt.

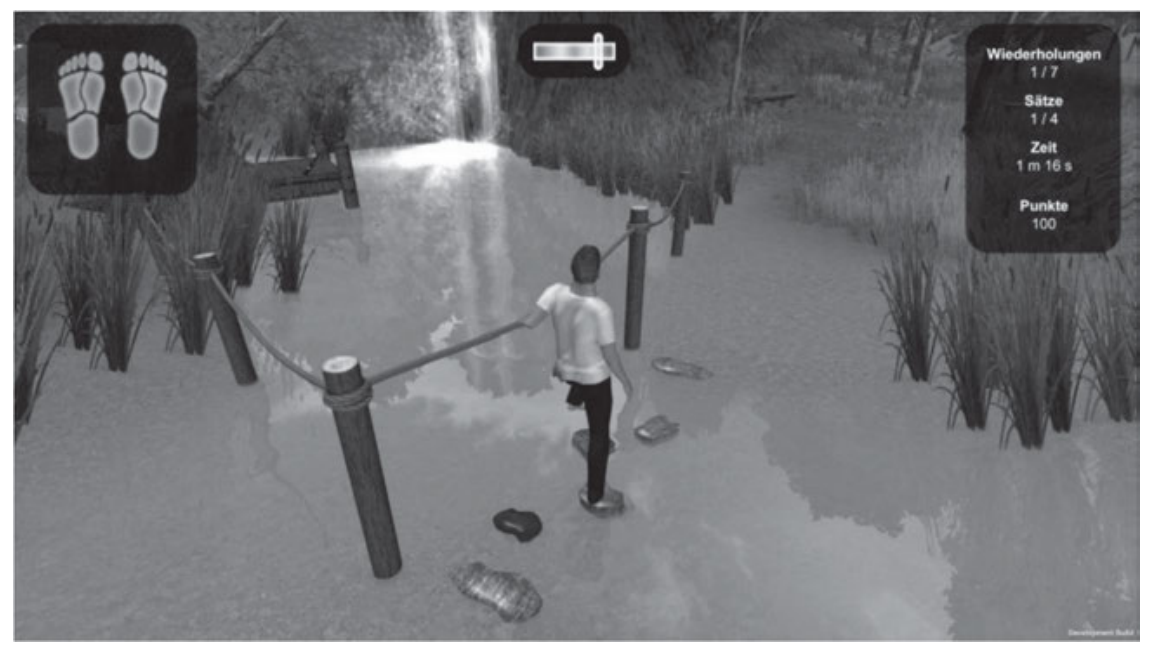

Abbildung 2: Überqueren eines Baches über Steine

Für die spielerische Umsetzung der Gangschule schlüpft der Patient in die Rolle eines Gärtners, der sich vor einem Fluss befindet. Innerhalb des Flusses befinden sich Steine, die einen Weg darstellen und zum Überqueren genutzt werden sollen. Die Wollie-Action wird symbolisiert durch ein Seil, das in Armhöhe über den Fluss führt und bei Bedarf von dem Patienten zur festhaltenden Unterstützung genutzt werden kann. Ziel des Spiels ist es, den Bach Schritt 
für Schritt zu überqueren ohne an dem Seil zu ziehen oder zu drücken. Wird eine bestimmte Kraft auf die Wollie-Action und somit auf das Seil ausgeübt, färbt sich dieses innerhalb eines Toleranzbereiches gelb und bei Überschreiten dieses Wertes rot ein. Somit wird dem Patienten als Feedback-Mechanismus signalisiert, dass er mehr auf sein Gleichgewicht achten sollte. Einstellbare Übungsparameter durch den betreuenden Therapeuten beziehen sich hierbei auf die Grenzwerte der erlaubten Krafteinwirkung auf die Wollie-Action (Gleichgewicht), auf die Größe der Steine, deren Abstände und somit auch auf die Anzahl (Beweglichkeit, Schrittlänge, Stellung der Füße zueinander, gleichmäßige Druckverteilung und Abrollbewegung). Jeder gesetzte Schritt durch den Patienten trifft bei Einsatz des richtigen Beins einen Stein unabhängig davon, ob die Schrittlänge den Vorgaben entspricht. Das Einfärben der Steine in die Farben grün, gelb oder rot zeigt dem Patienten an, wie erfolgreich der Schritt den eingestellten Übungsparametern entspricht.

\section{2 Übungen am Wandschienen-System Wollie-Action}

Zur Aktivierung der oberen Extremitäten wird insbesondere das Wand-Sensor-System eingesetzt. Je nach Übungsziel werden die Pads der Wollie-Action geschoben und gedrückt oder gezogen und dadurch die Arme, der Oberkörper und der Rumpf des Patienten gekräftigt sowie die Stabilisation der Schultern trainiert. Parameter wie Koordination, Beweglichkeit und mögliche Belastungsintensität des Oberkörpers durch Messung der Dauer der aufgebauten isometrischen Spannung durch anhaltendes drücken oder ziehen können innerhalb dieser Trainingsszenarien bewertet werden. Auch die gleichmäßige Kraftverteilung durch einen Rechts-Links-Vergleich wird durch den Einsatz der Wollie-Action ermöglicht. Durch Hinzunahme der Schuhsensorik kann zudem die gleichmäßige Lastverteilung auf den Fußsohlen gemessen und analysiert werden. Auf Basis der Daten der optischen Sensorik werden die Körperhaltung, der achsengerechte Stand sowie der Abstand zum Wandschienensystem und somit die Armstellung des Trainierenden kontrolliert. 


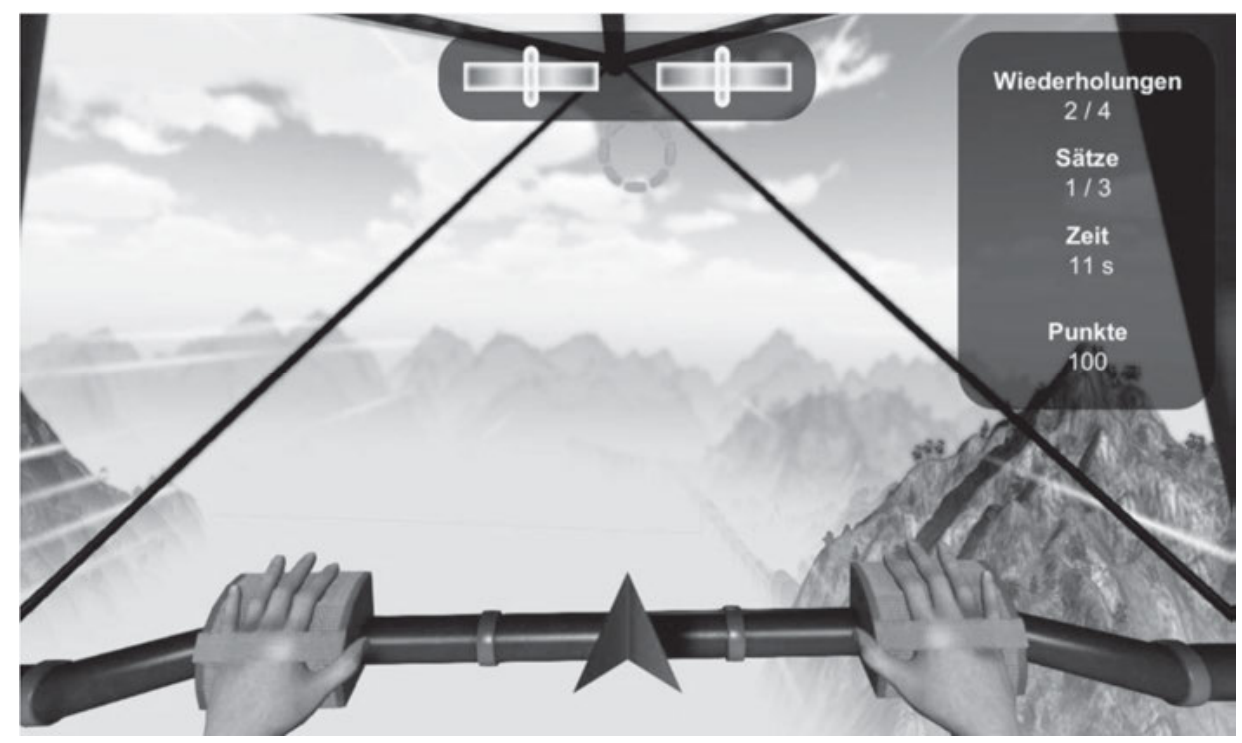

Abbildung 3: Drachenfliegen

Zur Aktivierung der oberen Extremitäten, insbesondere der Arme und der Schultern, wird der Patient zum Drachenflieger und befindet sich in der Luft über einer weitläufigen Landschaft. Ziel ist es nun durch Ringe zu fliegen, die nach und nach in unterschiedlicher Höhe am Horizont erscheinen. Indem die Pads der Wollie-Action entsprechend lange gedrückt oder gezogen werden, wird die Flughöhe beeinflusst. Die Richtung wird bestimmt durch Schieben der Pads entlang der Schienen des Wand-Systems. Die erforderliche Bewegungsrichtung wird durch das Einblenden von Pfeilen im Voraus angekündigt, so dass der Patient genug Zeit hat rechtzeitig zu reagieren. Übungsparameter können dabei durch den betreuenden Therapeuten individuell eingestellt werden und beziehen sich im Kontext dieses Trainingsszenarios auf die Frequenz und Position der auftretenden Ringe (Bewegungs- und Kraftintensität), die Geschwindigkeit des Gleiters (Reaktionszeit / Koordination) und die Anzahl der Ringe, die durchflogen werden müssen. Die Einfärbung des aktuell angeflogenen Rings kennzeichnet, wie erfolgreich dieser entsprechend der eingestellten Parameter getroffen wird.

\section{Ausblick}

Gegenwärtig befindet sich das Projekt in der Abschlussphase. Nach der Etablierung der technischen Infrastruktur für die Zusammenführung, Weiterleitung und Analyse der Sensorsignale, der Ausgestaltung des Interaktionskonzeptes und der Entwicklung der multimodalen Benutzeroberflächen für drei konkrete Bewegungsübungen erfolgt nun die Planung der Systemevaluation im Reha-Zentrum Lübben. Entwicklungsbegleitend wurde im Fraunhofer Institut FOKUS ein Therapieraum eingerichtet. Ab Mitte des Jahres wird ein weiterer funktionsfähiger Therapieraum in Lübben bereitstehen, so dass bis zum Ende des Jahres 2015 eine 
Evaluation des Gesamtsystems durchgeführt werden kann. Hierbei wird der Therapie-Server primär von den Therapeuten beurteilt und für die Evaluation des Therapie-Clients sind Patientenbefragungen geplant. Nach einer positiven Evaluation sollen Gespräche mit dem Kostenträger aufgenommen werden, das Konzept eines multimodalen, telemedizinisch assistierten Therapieraumes in die Versorgungspraxis zu überführen.

\section{Schlussteil}

\section{Literaturverzeichnis}

Aichner, F. and Holzer, E. (2013). Schlaganfall: Vorsorge, Behandlung und Nachsorge - Ein Ratgeber für Gesunde, Patienten und Angehörige. Wien: Springer Verlag.

Botella, C., Etchemendy, E., Castilla, D., Banos, R.M., Garcia-Palacios, A., Quero, S., Alcaniz, M. \& Lozano, J.A. 2009. An e-health system for the elderly (Butler Project): A pilot study on acceptance and satisfaction. In Liebert, M.A. (Hrsg.): CyberPsychology \& Behavior 12(3). S. 255-262

Cyarto, E.V., Brown, W.J., Marshall, A.L. \& Trost, S.G. (2008). Comparative effects of home- and group-based exercise on balance confidence and balance ability in older adults: cluster randomized trial. Gerontology 54(5), S. 272-280.

Eiff, W. von \& Schüring, S. (2011). Medizinische Rehabilitation: Kürzere Akut-Verweildauern erhöhen Aufwand in der Reha. Deutsches Ärzteblatt 108(21), S. 20

Hamdi, O., Chalouf, M.A., Ouattara, D. \& Krief, F. (2014). eHealth: Survey on research projects, comparative study of telemonitoring architectures and main issues. Journal of Network and Computer Applications 46, S. 100-112

Hermens, H.J. \& Vollenbroek-Hutten, M.M.R. (2008). Towards remote monitoring and remotely supervised training. Journal of Electromyography and Kinesiology: Official Journal of the International Society of Electrophysiological Kinesiology 18(6), S. 908-919

ISO/IEC/IEEE Health informatics--Personal health device communication--Part 20601: Application profile--Optimized exchange protocol (2010). ISO/IEEE 11073-20601:2010(E), S. 1-208

John, M., Klose, S., Kock, G., Jendreck, M., Feichtinger, R., Henning, B., Reithinger, N., Kiselev, J., Gövercin, M., Steinhagen-Thiessen, E., Kausch, S., Polak, M. \& Irmscher, B. 2012. SmartSenior's Interactive Trainer - Development of an Interactive System for a Home-Based Fall-Prevention Training for Elderly People. In Wichert, R \& Eberhardt, B. (Hrsg.): Ambient Assisted Living 5, S. 305-316

Kliem, A., Boelke, A., Grohnert, A. \& Traeder, N. 2014. Self-adaptive middleware for ubiquitous medical device integration. In IEEE (Hrsg.): e-Health Networking, Applications and Services (Healthcom), 2014 IEEE 16th International Conference on, S. 298-304

Koczula, G., Schultz, C \& Gövercin, M. 2012. Die Rolle von technologiebasierten Assistenzsystemen bei der ganzheitlichen Versorgung pflegebedürftiger Patienten - Herausforderungen einer flächendeckenden Implementierung. In Shire, K.A. \& Leinmeister, J.M. (Hrsg.): Technologiegestützte Dienstleistungsinnovation in der Gesundheitswirtschaft. Wiesbaden: Gabler Verlag, S. 31-55

Körtke, H., Feige, T. Gräfe, B. \& Secer, S. (2012). Akzeptanz der Telemedizin. In Technik für ein selbstbestimmtes Leben (5. Deutscher AAL-Kongress). Berlin: VDE Verlag GmbH 
Welsch, N. (2015). Leben ohne Tod? Forscher besiegen das Altern. Berlin, Heidelberg: Springer Spectrum

\section{Kontaktinformationen}

Anne Grohnert

Komplexe und Verteilte IT Systeme (CIT), Technische Universität Berlin

Einsteinufer 17, 10587 Berlin

anne.grohnert@tu-berlin.de

Anett Bölke

Komplexe und Verteilte IT Systeme (CIT), Technische Universität Berlin Einsteinufer 17, 10587 Berlin

anett.boelke@tu-berlin.de

Benny Häusler

Kompetenzzentrum E-HEALTH, Fraunhofer FOKUS

Kaiserin-Augusta-Allee 31, 10589 Berlin

benny.haeusler@fokus.fraunhofer.de

Boris Irmscher

Nuromedia GmbH, Schaafenstr. 25, 50676 Köln

boris.irmscher@nuromedia.com

Michael John

Kompetenzzentrum E-HEALTH, Fraunhofer FOKUS

Kaiserin-Augusta-Allee 31, 10589 Berlin

michael.john@fokus.fraunhofer.de

Andreas Kliem

Komplexe und Verteilte IT Systeme (CIT), Technische Universität Berlin

Einsteinufer 17, 10587 Berlin

andreas.kliem@tu-berlin.de

Gerd Kock

Kompetenzzentrum E-HEALTH, Fraunhofer FOKUS

Kaiserin-Augusta-Allee 31, 10589 Berlin

gerhard.kock@fokus.fraunhofer.de

Jens Piesk

Nuromedia GmbH

Schaafenstr. 25, 50676 Köln

jens.piesk@nuromedia.com

Marco Polak

Nuromedia GmbH

Schaafenstr. 25, 50676 Köln

boris.irmscher@nuromedia.com 